\title{
Complex and Transitive Synchronization in a Frustrated System of Calling Frogs
}

$\operatorname{AUTHOR}(S):$

Aihara, Ikkyu; Takeda, Ryu; Mizumoto, Takeshi; Otsuka, Takuma; Takahashi, Toru; Okuno, Hiroshi G; Aihara, Kazuyuki

\section{CITATION:}

Aihara, Ikkyu ...[et al]. Complex and Transitive Synchronization in a

Frustrated System of Calling Frogs. Physical Review E 2011, 83: 031913.

\section{ISSUE DATE:}

2011-03-21

URL:

http://hdl.handle.net/2433/187383

RIGHT:

(C2011 American Physical Society 
PHYSICAL REVIEW E 83, 031913 (2011)

\title{
Complex and transitive synchronization in a frustrated system of calling frogs
}

\author{
Ikkyu Aihara \\ Department of Physics, Graduate School of Sciences, Kyoto University, Kyoto, Japan \\ Ryu Takeda, Takeshi Mizumoto, Takuma Otsuka, Toru Takahashi, and Hiroshi G. Okuno \\ Department of Intelligence Science and Technology, Graduate School of Informatics, Kyoto University, Kyoto, Japan \\ Kazuyuki Aihara \\ Institute of Industrial Science, University of Tokyo, Tokyo, Japan \\ (Received 10 October 2010; revised manuscript received 8 December 2010; published 21 March 2011; corrected 20 April 2011)

\begin{abstract}
This letter reports synchronization phenomena and mathematical modeling on a frustrated system of living beings, or Japanese tree frogs (Hyla japonica). While an isolated male Japanese tree frog calls nearly periodically, he can hear sounds including calls of other males. Therefore, the spontaneous calling behavior of interacting males can be understood as a system of coupled oscillators. We construct a simple but biologically reasonable model based on the experimental results of two frogs, extend the model to a system of three frogs, and theoretically predict the occurrence of rich synchronization phenomena, such as triphase synchronization and 1:2 antiphase synchronization. In addition, we experimentally verify the theoretical prediction by ethological experiments on the calling behavior of three frogs and time series analysis on recorded sound data. Note that the calling behavior of three male Japanese tree frogs is frustrated because almost perfect antiphase synchronization is robustly observed in a system of two male frogs. Thus, nonlinear dynamics of the three-frogs system should be far from trivial.
\end{abstract}

DOI: 10.1103/PhysRevE.83.031913

PACS number(s): 87.23.-n, 05.45.Xt

\section{INTRODUCTION}

Synchronization is ubiquitous in real systems, and has been attracting a great deal of attention in physics as well as in many other disciplines since the seminal work on Huygens' clocks [1]. In addition, theoretical studies revealed plausible synchronization mechanisms for accomplishing cooperative phenomena in the real world, including phase-oscillator models [1], a phase-reduction method for a general class of noisy oscillators [2], feedback control [3] and coevolution of phases and coupling strengths [4] in coupled oscillator systems, and a general chemotactic model of oscillators [5]. However, those theoretical studies mainly focused on in-phase synchronization and nonlinear dynamics of antiphase synchronization in a coupled system had not been sufficiently investigated [6]. In particular, antiphase synchronization is widely observed in acoustic communications by a pair of living beings, including birds, mammals, crickets, and frogs [7-9]; such alternating calling behavior plays an important role in their communications to transmit sound information included in the interacting calling behavior by mutually avoiding overlaps of their calls.

From theoretical points of view, antiphase synchronization between a pair raises an interesting fundamental problem in a coupled system with many oscillators [6]; antiphase synchronization between a pair cannot be realized in every pair of three oscillators because, when two pairs of the three synchronize in antiphase, respectively, the remaining third pair must synchronize in-phase. Thus, such a system of more than two oscillators is generally frustrated. Note that a similar situation of the frustration can be seen in antiferromagnetic spin systems [10]. From the viewpoint of frustration, however, the coupled-oscillator systems show an important difference from the spin systems; while the state of a spin is limited to up or down [10], that of a phase oscillator is described with a phase variable $\theta \in \mathbb{S}^{1}[6,9]$.

To clarify possibly complex behavior in such a frustrated system of coupled phase oscillators, it is reasonable to first investigate the simplest case of the frustration. In this paper, we theoretically and experimentally study the spontaneous calling behavior of three male Japanese tree frogs Hyla japonica shown in Fig. 1(a) [6,9] as a frustrated system of living beings because our experimental observations revealed that, while a single male Japanese tree frog calls nearly periodically, a couple of males generate robust antiphase synchronization [9] [see Figs. 1(b) and 1(c)].

The paper is organized as follows. In Sec. II, we construct a possible phase oscillator model representing interactive calling behavior by three frogs and theoretically predict synchronization phenomena. In Sec. III, we empirically verify the theoretical prediction shown in Sec. II by ethological experiments and time series analysis. In Sec. IV, we discuss the relationship between the modeling and experimental results, biological implications by the model analysis, and possible biological meanings of synchronized behavior by frogs.

\section{MATHEMATICAL MODELING}

To predict the possible synchronized behavior of three Japanese tree frogs, we first construct a simple but biologically reasonable model based on the experimental results of two frogs shown in Fig. 1(c) that two frogs robustly synchronize in antiphase [9]. Let us model calling behavior of the two frogs $\alpha$ and $\beta[1,9]$, as follows:

$$
\frac{d \theta_{\alpha}}{d t}=\omega_{\alpha}+K\left\{\sin \left(\theta_{\alpha}-\theta_{\beta}\right)-\gamma \sin \left[2\left(\theta_{\alpha}-\theta_{\beta}\right)\right]\right\},
$$


(a)

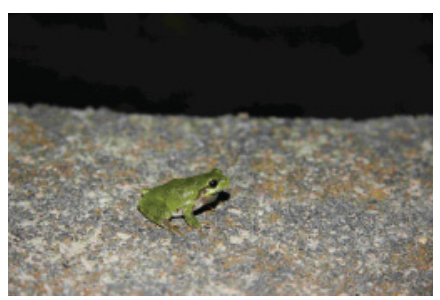

(b)

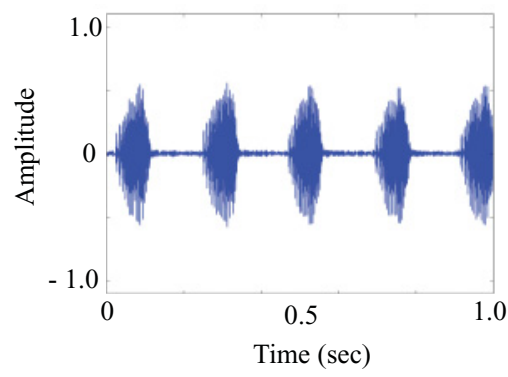

(c)

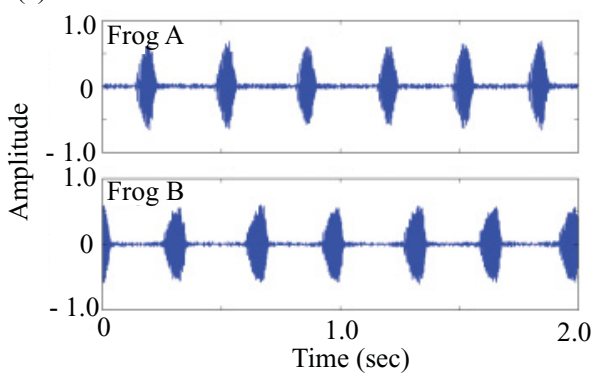

FIG. 1. (Color online) Calling behavior of one or two frogs. (a) Japanese tree frog Hyla japonica. (b) Periodic calling behavior of a single male frog. (c) Antiphase synchronization of two frogs. Figs. 1(b) and 1(c) represent sound data of a single frog and those of two frogs, respectively.

$$
\frac{d \theta_{\beta}}{d t}=\omega_{\beta}+K\left\{\sin \left(\theta_{\beta}-\theta_{\alpha}\right)-\gamma \sin \left[2\left(\theta_{\beta}-\theta_{\alpha}\right)\right]\right\},
$$

where phases $\theta_{\alpha}$ and $\theta_{\beta}$ represent the call timings of the frogs $\alpha$ and $\beta$ (i.e., when $\theta_{\alpha}=0$, we consider that the frog $\alpha$ calls), $\omega_{\alpha}$ and $\omega_{\beta}$ are the natural frequencies of respective frogs (i.e., $2 \pi / \omega_{\alpha}$ and $2 \pi / \omega_{\beta}$ are the intrinsic intercall intervals [8]), $K$ is the positive coupling strength between two frogs due to their acoustic communication, and $\gamma$ is a positive parameter which represents the effect of the second-order component in the interaction term or changes the stability between the antiphase and in-phase states [9]. Our experimental observations on the interactive calling behavior of two frogs show that, while antiphase synchronization of two frogs is robustly observed, in-phase synchronization is detected only transiently [9]. Next, we introduce suitable parameter values in Eqs. (1) and (2) to explain the experimental results of two frogs. First, it is experimentally shown that the intrinsic frequencies $\omega_{\alpha}$ and $\omega_{\beta}$ tend to take close values under the same experimental condition [9]. Hence we assume $\omega_{\alpha}=\omega_{\beta}$ for the sake of simplicity. Note that, under this first assumption, both antiphase and in-phase synchronization states (i.e., $\phi=\pi$ and $\phi=0$ with $\phi \equiv \theta_{\alpha}-\theta_{\beta}$ ) become equilibrium points, namely $\left.\frac{d \phi}{d t}\right|_{\phi=\pi}=0$ and $\left.\frac{d \phi}{d t}\right|_{\phi=0}=0$. In addition, $\gamma$ is assumed to take a positive value of less than 0.5 . The linear stability of the antiphase state and that of the in-phase state can be analyzed by $\left.\frac{\partial}{\partial \phi} \frac{d \phi}{d t}\right|_{\phi=\pi}=-2 K(1+2 \gamma)$ and $\left.\frac{\partial}{\partial \phi} \frac{d \phi}{d t}\right|_{\phi=0}=2 K(1-2 \gamma)$, respectively. When $\omega_{\alpha}=\omega_{\beta}$ and $0 \leqslant \gamma<0.5$, the former is negative and the latter positive. Therefore, under these two assumptions of $\omega_{\alpha}=\omega_{\beta}$ and $0 \leqslant \gamma<0.5$, the experimental results on a two-frogs system [9] is qualitatively explained; namely, antiphase synchronization of two frogs is represented as a stable equilibrium and the in-phase synchronization is described as an unstable one.

Extending Eqs. (1) and (2) to a system of three coupled oscillators, we model the spontaneous calling behavior of three frogs as follows:

$$
\frac{d \theta_{i}}{d t}=\omega_{i}+\sum_{j=A, B, C} K_{i j}\left\{\sin \left(\theta_{i}-\theta_{j}\right)-\gamma \sin \left[2\left(\theta_{i}-\theta_{j}\right)\right]\right\}
$$

where $\theta_{i}(i=A, B, C)$ represents the timing of successive calls by the frog, $i, \omega_{i}$ is its intrinsic frequency, and $K_{i j}$ is the positive coupling strength between the frogs $i$ and $j$ with $K_{i j}=$ $K_{j i}$ [6]. Let us consider suitable parameter values in Eq. (3) which qualitatively explain the experimental situation shown in Fig. 2(a), and perform bifurcation analysis with bifurcation parameters $\gamma$ and $K_{A C}$ to theoretically predict the possible synchronized calling behavior of three frogs. First, we assume $\omega_{A}=\omega_{B}=\omega_{C}$ for the same reason as in a system of two frogs. Second, since male frogs interact through sounds and (a)

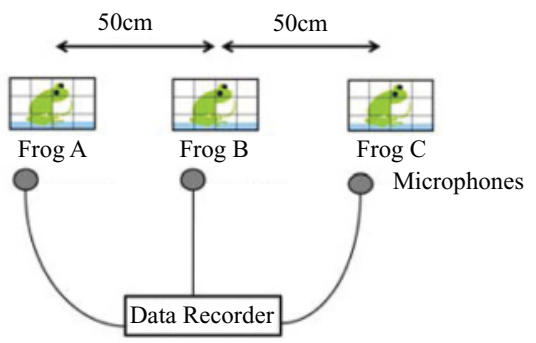

(b)

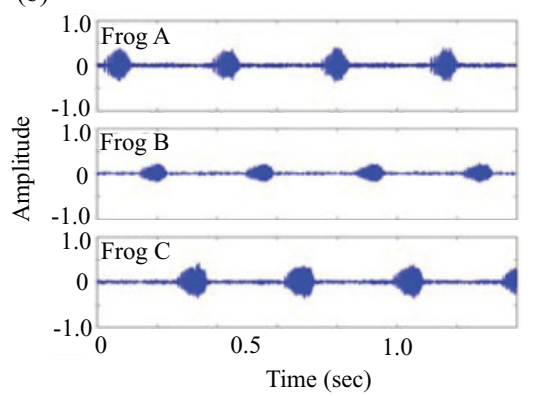

(c)

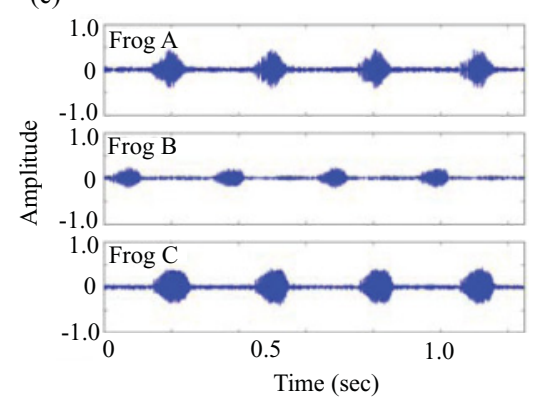

FIG. 2. (Color online) Calling behavior of three frogs. (a) Schematic diagram describing the experimental situation; three frogs were put in cages, respectively, and set along a straight line at a distance of $50 \mathrm{~cm}$. Spontaneous calling behavior was recorded by three microphones placed close to each frog. (b) Triphase synchronization of $\mathrm{A} \rightarrow \mathrm{B} \rightarrow \mathrm{C}$; the three frogs $\mathrm{A}, \mathrm{B}$, and $\mathrm{C}$ call in turns with the phase difference of $2 \pi / 3$. (c) $1: 2$ antiphase synchronization of $B$ vs AC; the two frogs $A$ and $C$ synchronize in-phase of 0 , and the remaining one of frog $B$ synchronizes in antiphase of $\pi$ with the others. In Figs. 2(b) and 2(c), sound data of respective frogs are separated by independent component analysis. 
the distance between frogs $A$ and $B$ is identical to that between frogs $B$ and $C$ in the experiments [see Fig. 2(a)], we assume that the adjacent pairs interact with the same strength (i.e., $K_{A B}=K_{B C}=1.0$, for simplicity). Then, we vary bifurcation parameters $\gamma$ and $K_{A C}$ within $0 \leqslant \gamma<0.5$ and $0 \leqslant K_{A C}<$ 1.0 , and calculate stable phase differences of $\phi_{A B} \equiv \theta_{A}-\theta_{B}$ and $\phi_{A C} \equiv \theta_{A}-\theta_{C}$. The former assumption of $0 \leqslant \gamma<0.5$ is consistent with that in the model analysis on a two-frogs system. In addition, since the distance between frogs $A$ and $C$ is longer than that between frogs $A$ and $B$ as shown in Fig. 2(a), $K_{A C}$ is assumed to be less than $K_{A B}=1.0$.

The results of the bifurcation analysis are summarized in Fig. 3. Note that, throughout the model analysis, we set the error bound of $\pi / 6$ for detection on synchronization modes, the same as in the time series analysis performed on experimental data where the phase differences between frogs (i.e., $\phi_{A B}$ and $\phi_{A C}$ ) can fluctuate even in almost synchronized states. As a result, it is theoretically predicted that, depending on values of $K_{A C}$ and $\gamma$, two types of triphase synchronization $(\mathrm{A} \rightarrow \mathrm{B} \rightarrow \mathrm{C}$ and $\mathrm{A} \rightarrow \mathrm{C} \rightarrow \mathrm{B})$ and three types of $1: 2$ antiphase synchronization (A versus $\mathrm{BC}, \mathrm{B}$ versus $\mathrm{AC}$, and $\mathrm{C}$ versus $A B$ ) can be observed under the experimental situation shown in Fig. 2(a). For example, triphase synchronization of $\mathrm{A} \rightarrow \mathrm{B} \rightarrow \mathrm{C}$ means $\phi_{A B} \simeq 2 \pi / 3$ and $\phi_{A C} \simeq 4 \pi / 3 ; 1: 2$ antiphase synchronization of $\mathrm{A}$ versus $\mathrm{BC}$ means that, while the two oscillators $B$ and $C$ synchronize in nearly in-phase, the remaining one $A$ synchronize in nearly antiphase with $B$ and $C$. Details of the synchronization properties are explained in the caption of Fig. 3.

\section{EXPERIMENTS}

We experimentally verified the model prediction. Spontaneous calling behavior of three male Japanese tree frogs was recorded with microphones placed close to each frog, as shown in Fig. 2(a). The experiments were carried out a total of 44 times on May and June, both in 2008 and 2009, and four trials where three frogs successively called were obtained corresponding to Data (1)-(4) in Figs. 4(b)-4(e) and Table I. In those experiments, the recording time for each trial was about 4 hours. Recorded sound data were analyzed to separate individual calling signals by the independent component analysis (ICA) [11]. During the calling behavior of three frogs, various types of synchronization were robustly observed as typically shown in Figs. 2(b) and 2(c) (listen also to supplementary sound files [12]): The first one in Fig. 2(b) represents the triphase synchronization of $\mathrm{A} \rightarrow \mathrm{B} \rightarrow \mathrm{C}$ that the three frogs $\mathrm{A}, \mathrm{B}$, and $\mathrm{C}$ called in turns with the phase difference of almost $2 \pi / 3$; the second one in Fig. 2(c) is $1: 2$ antiphase synchronization of $B$ versus $A C$ that two of the three frogs (frogs A and C) synchronized almost in-phase, while the remaining one (frog B) synchronized almost in antiphase with the others.

Separated sound signals were analyzed to estimate the phase differences of three frogs (i.e., $\phi_{A B} \equiv \theta_{A}-\theta_{B}$ and $\left.\phi_{A C} \equiv \theta_{A}-\theta_{C}\right)$ according to the methods shown in the supplemental materials [12]. Consequently, we stably observed various types of synchronized behavior and switching phenomena as shown in Figs. 4(b)-4(e), including three types (a)

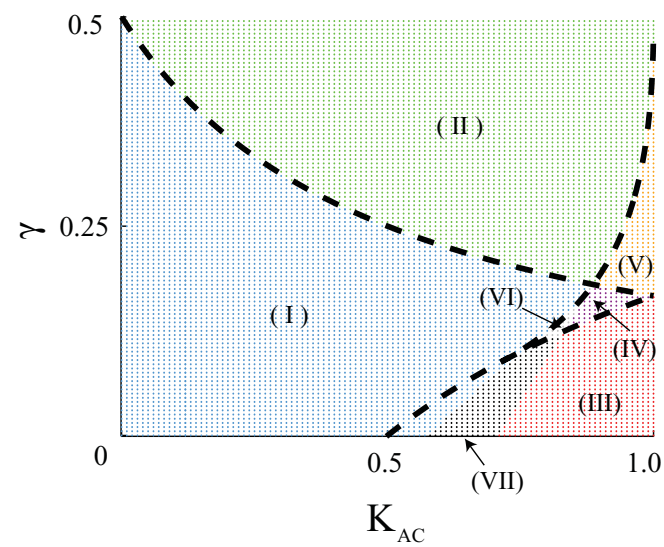

(b)

$$
\mathrm{K}_{\mathrm{AC}}=1.0
$$

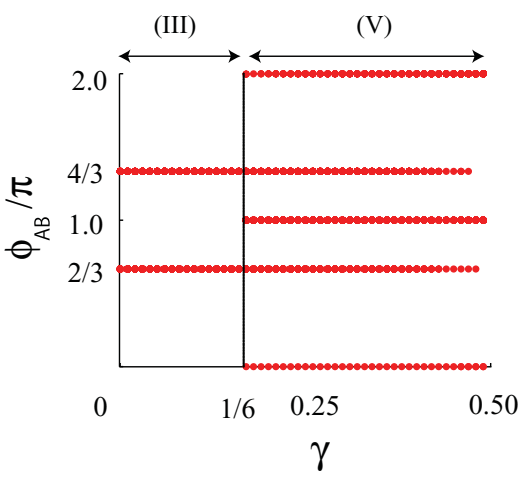

FIG. 3. (Color) Bifurcation analysis on the model of Eq. (3) with the bifurcation parameters $K_{A C}$ and $\gamma$. (a) Phase diagram of synchronization states on the $K_{A C}-\gamma$ plain. Here, we set the error bound of $\pi / 6$ to detect synchronization modes and compare with the experimental data. Region (I), depicted with blue dots, represents parameter values at which the 1:2 antiphase synchronization of $\left(\phi_{A B}, \phi_{A C}\right) \simeq(\pi, 0)$ is stable. Region (II) with green dots is the multistable state for three types of 1:2 antiphase synchronization $\left(\phi_{A B}, \phi_{A C}\right) \simeq(\pi, 0),(0, \pi)$, and $(\pi, \pi)$. Region (III) with red dots is for two types of triphase synchronization $\left(\phi_{A B}, \phi_{A C}\right) \simeq(2 \pi / 3,4 \pi / 3)$ and $(4 \pi / 3,2 \pi / 3)$. Region (IV) with purple dots is for one of the 1:2 antiphase synchronization $\left(\phi_{A B}, \phi_{A C}\right) \simeq(\pi, 0)$ and two types of triphase synchronization. Region (V) with yellow dots is for three types of 1:2 antiphase synchronization and two types of triphase synchronization. Region (VI) with gray dots is for one of the 1:2 antiphase synchronization $\left(\phi_{A B}, \phi_{A C}\right) \simeq(\pi, 0)$ and the synchronization states different from both triphase and 1:2 antiphase synchronization. Region (VII) with black dots is for the synchronization states different from both the triphase and 1:2 antiphase synchronization. Dotted lines give exact bifurcation sets obtained by the bifurcation stability analysis [6]. (b) Bifurcation structure along $K_{A C}=1.0$ in Fig. 3(a), where the vertical axis represents a stable phase difference of $\phi_{A B}$. While two types of triphase synchronization with $\left(\phi_{A B}, \phi_{A C}\right)=(2 \pi / 3,4 \pi / 3)$ and $(4 \pi / 3,2 \pi / 3)$ are bistable in $0 \leqslant \gamma<\gamma_{*}$, two types of triphase synchronization and three types of 1:2 antiphase synchronization are multistable in $\gamma_{*} \leqslant \gamma<0.5$ with $\gamma_{*}=1 / 6$; the former state corresponds to Region (III) in Fig. 3(a), and the latter corresponds to region (V). 


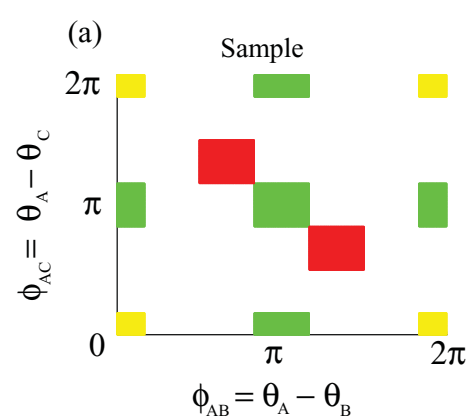

(b)

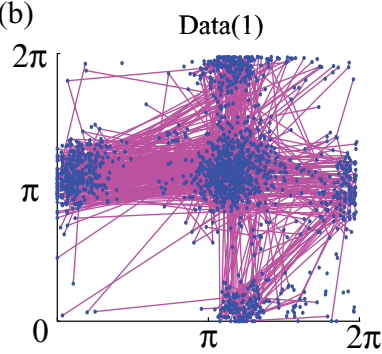

(c) $\quad \operatorname{Data}(2)$

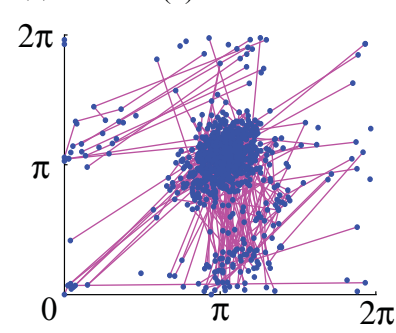

(d)

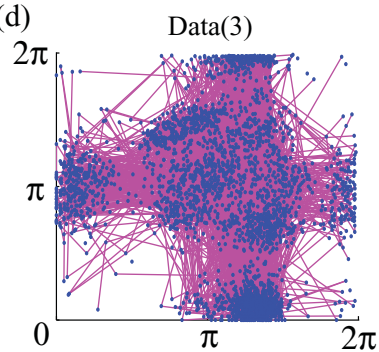

(e)

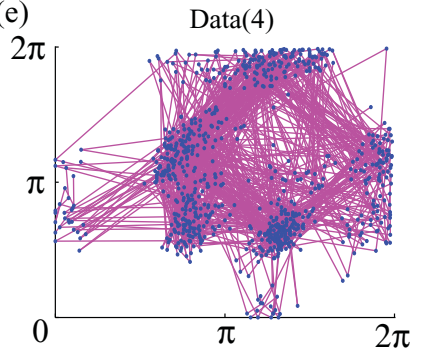

FIG. 4. (Color) Synchronization and switching dynamics experimentally observed in a three-frogs system. (a) Definition of synchronization states on the phase plain of $\phi_{A B}$ (the abscissa) and $\phi_{A C}$ (the ordinate), setting the error bound of $\pi / 6$. Regions of triphase synchronization, 1:2 antiphase synchronization, and in-phase synchronization are shown with red, green, and yellow rectangles, respectively. (b-e) Plots of the phase differences $\phi_{A B}$ and $\phi_{A C}$ obtained from four experimental trials. Note that the phase plots shown by blue dots are localized not around the in-phase state but around the triphase and 1:2 antiphase states. Transitions between different states are indicated by pink lines.

of 1:2 antiphase synchronization (A versus BC, B versus $A C$, and $C$ versus $A B$ ) and two types of triphase synchronization $(\mathrm{A} \rightarrow \mathrm{B} \rightarrow \mathrm{C}$ and $\mathrm{A} \rightarrow \mathrm{C} \rightarrow \mathrm{B}$ ); Fig. 4(b) represents the occurrence of three types of 1:2 antiphase synchronization (A versus $\mathrm{BC}$, B versus $\mathrm{AC}$, and $\mathrm{C}$ versus $\mathrm{AB}$ ), Fig. 4(c) represents that of mainly only one of the 1:2 antiphase synchronization
(A versus BC), Fig. 4(d) shows that of the three types of 1:2 antiphase synchronization (A versus $\mathrm{BC}, \mathrm{B}$ versus $\mathrm{AC}$, and $C$ versus $A B)$ and the two types of triphase synchronization $(\mathrm{A} \rightarrow \mathrm{B} \rightarrow \mathrm{C}$ and $\mathrm{A} \rightarrow \mathrm{C} \rightarrow \mathrm{B})$, and Fig. 4(e) shows that of the two types of triphase synchronization $(\mathrm{A} \rightarrow \mathrm{B} \rightarrow \mathrm{C}$ and $\mathrm{A} \rightarrow \mathrm{C} \rightarrow \mathrm{B}$ ) and one of the 1:2 antiphase synchronization

TABLE I. Summary of synchronized behavior obtained from the experimental data of calling frogs. Total calling durations of a single frog, two frogs, and three frogs, and temporal sums of detected synchronization modes are shown for four experimental trials (see Ref. [12]). As for the calling of two frogs, antiphase synchronization is robustly observed, but in-phase synchronization is rarely detected. During the calling of three frogs, triphase synchronization and 1:2 antiphase synchronization can be stably observed compared with the in-phase synchronization.

\begin{tabular}{|c|c|c|c|c|c|}
\hline & Total & Antisync & Trisync & 1:2 Antisync & In-Sync \\
\hline \multicolumn{6}{|l|}{ Data (1) } \\
\hline One Frog & $173.1 \mathrm{~s}$ & & & & \\
\hline Two Frogs & $378.2 \mathrm{~s}$ & $138.7 \mathrm{~s}(36.6 \%)$ & & & $11.7 \mathrm{~s}(3.1 \%)$ \\
\hline Three Frogs & $660.8 \mathrm{~s}$ & & 34.7 s $(5.2 \%)$ & $64.0 \mathrm{~s}(9.6 \%)$ & $0 \mathrm{~s}(0 \%)$ \\
\hline \multicolumn{6}{|l|}{ Data (2) } \\
\hline One Frog & $732.2 \mathrm{~s}$ & & & & \\
\hline Two Frogs & $1416.4 \mathrm{~s}$ & $595.3 \mathrm{~s}(42.0 \%)$ & & & $39.6 \mathrm{~s}(2.8 \%)$ \\
\hline Three Frogs & $298.3 \mathrm{~s}$ & & $15.5 \mathrm{~s}(5.2 \%)$ & 60.4 s $(20.2 \%)$ & $2.5 \mathrm{~s}(0.8 \%)$ \\
\hline \multicolumn{6}{|l|}{ Data (3) } \\
\hline One Frog & $104.8 \mathrm{~s}$ & & & & \\
\hline Two Frogs & $1088.2 \mathrm{~s}$ & $396.7 \mathrm{~s}(36.4 \%)$ & & & $41.3 \mathrm{~s}(3.8 \%)$ \\
\hline Three Frogs & $1265.8 \mathrm{~s}$ & & $91.4 \mathrm{~s}(7.2 \%)$ & $33.2 \mathrm{~s}(2.6 \%)$ & $0 \mathrm{~s}(0 \%)$ \\
\hline \multicolumn{6}{|l|}{ Data (4) } \\
\hline One Frog & $101.7 \mathrm{~s}$ & & & & \\
\hline Two Frogs & $976.7 \mathrm{~s}$ & $621.1 \mathrm{~s}(63.5 \%)$ & & & $22.4 \mathrm{~s}(2.2 \%)$ \\
\hline Three Frogs & $350.6 \mathrm{~s}$ & & $52.8 \mathrm{~s}(15.0 \%)$ & $4.2 \mathrm{~s}(1.1 \%)$ & $0 \mathrm{~s}(0 \%)$ \\
\hline
\end{tabular}


(A versus BC). Moreover, the (pink) solid lines in Figs. 4(b)-4(e) show the existence of complex switching dynamics in this frogs' system.

Moreover, we categorize the obtained phase differences $\phi_{A B}$ and $\phi_{A C}$ into synchronization modes to estimate the stability or durations of respective modes as shown in Table I (see also supplemental materials [12]). During the calling behavior of two frogs, while antiphase synchronization is robustly observed, in-phase synchronization is rarely detected. In addition, during the total recording time of three frogs, triphase synchronization and 1:2 antiphase synchronization can be robustly observed compared with the in-phase synchronization by all three frogs.

\section{DISCUSSION}

We show that mathematical modeling with Eq. (3) qualitatively explains the experimental results on the synchronized calling behavior of three frogs summarized in Figs. 4(b)-4(e) assuming $\omega_{A}=\omega_{B}=\omega_{C}$ and $0 \leqslant \gamma<0.5$. The multistability between three types of 1:2 antiphase synchronization in Data (1), and the stability of one of the 1:2 antiphase synchronization (A versus BC) in Data (2) are qualitatively described in region (II) in Fig. 3(a). The multistability between two types of triphase synchronization and three types of 1:2 antiphase synchronization in Data (3), and that between two types of triphase synchronization and one of the 1:2 antiphase synchronization (A versus BC) in Data (4) are reproduced in region (V) in Fig. 3(a). Note that, although mathematical modeling in our previous study [6] gave a similar framework with only the first-order component in the interaction terms of Eq. (3), this cannot explain the complicated multistability as shown in Figs. 4(b)-4(e) with fixed parameter values. Since an experimental condition in respective trials is almost stationary, parameter values in model analysis should be constant for describing each datum. In this sense, mathematical modeling with Eq. (3) successfully explains complex synchronization phenomena by three frogs compared with our previous study [6]. From the viewpoint of mathematical modeling, however, it is an important future problem to clarify mechanisms responsible for the transitive and switching phenomena shown in Figs. 4(b)-4(e), by considering effects such as background noise [2] and time delay [13] as well as those of chaotic dynamics [14]; note that chaotic systems also generate antiphase synchronization [15].

Now, let us discuss the biological implications from the model analysis. The important point is that triphase synchronization of three frogs can be stably observed as shown in Data (3) and (4) and qualitatively explained with the model analysis by assuming the condition of $K_{A C} \simeq K_{A B}=K_{B C}$ such as regions (III), (IV), and (V) in Fig. 3(a). Since the coupling coefficient of $K_{i j}$ represents how strongly the frogs $i$ and $j$ interact, the condition of $K_{A C} \simeq K_{A B}=K_{B C}$ biologically implies that, during the interactive calling behavior of three frogs in the straight setting of Fig. 2(a), everyone can equally recognize the other two individuals and then triphase synchronization is realized. It should be noted that the similar calling property is reported in another species of frogs, Puerto Rican tree frogs; a single frog avoids acoustic overlap with two neighbors [16].

From an ethological point of view, it is important that our experiment clearly shows the existence of complex synchronized behavior by three male frogs to maintain their mutual intercall intervals each other (i.e., the triphase synchronization and 1:2 antiphase synchronization). Such alternating phenomena by three frogs would be biologically meaningful both for males and females to keep their own territories and localize calling males, the same as discussed in our previous study on a two-frogs system [9].

\section{ACKNOWLEDGMENTS}

This work was supported by JSPS Grant-in-Aid (Grant No.20-608), JSPS Grant-in-Aid for Exploratory Research, the JSPS FIRST Program, Honda Research Institute Japan, Co. Ltd., and Global COE Program in the Division of Physics and Astronomy, Kyoto University. We would like to thank T. Ohta, Y. Kuramoto, H. P. C. Robinson, H. Kitahata, Y. Kato, and T. Aoki, for their fruitful discussions, and also wish to thank K. Homma and A. Kitajima for their kind cooperation with the field research.
[1] Y. Kuramoto, Chemical Oscillations, Waves, and Turbulence (Springer-Verlag, Berlin, 1984); A. T. Winfree, J. Theor. Biol. 16, 15 (1967); A. Pikovsky, M. Rosenblum, and J. Kurths, Synchronization: A Universal Concept in Nonlinear Sciences. (Cambridge University Press, Cambridge, England, 2001).

[2] J. N. Teramae, H. Nakao, and G. B. Ermentrout, Phys. Rev. Lett. 102, 194102 (2009).

[3] I. Z. Kiss et al., Science 316, 1886 (2007).

[4] T. Aoki and T. Aoyagi, Phys. Rev. Lett. 102, 034101 (2009).

[5] D. Tanaka, Phys. Rev. Lett. 99, 134103 (2007).

[6] I. Aihara and K. Tsumoto, Math. Biosci. 214, 6 (2008).

[7] S. Yoshida and K. Okanoya, Cognitive Studies 12, 153 (2005).

[8] K. D. Wells, The Ecology and Behavior of Amphibians (University of Chicago Press, Chicago, 2007); H. C. Gerhardt and F. Huber, Acoustic Communication in Insects and Anurans (University of Chicago Press, Chicago, 2002).
[9] I. Aihara, Phys. Rev. E 80, 011918 (2009).

[10] H. T. Diep, Frustrated Spin Systems (World Scientific, Singapore, 2005).

[11] A. Hyvarinen, J. Karhunen, and E. Oja, Independent Component Analysis (Wiley Interscience, New York, 2001); H. Sawada, R. Mukai, and S. Araki, IEICE Transactions on Fundamentals of Electronics, Communications and Computer Sciences, E86-A, 505 (2003).

[12] See supplemental material at [http://link.aps.org/supplemental/ 10.1103/PhysRevE.83.031913] for sound files and experimental details.

[13] A. Takamatsu, T. Fujii, and I. Endo, Phys. Rev. Lett. 85, 2026 (2000).

[14] Y. Hirata et al., Chaos 20, 013117 (2010).

[15] L.-Y. Cao and Y.-C. Lai, Phys. Rev. E 58, 382 (1998).

[16] J. S. Brush and P. M. Narins, Anim. Behav. 37, 33 (1989). 